\title{
BMJ Open Feasibility and acceptability of e- PROMs data capture and feedback among patients receiving haemodialysis in the Symptom monitoring WIth Feedback Trial (SWIFT) pilot: protocol for a qualitative study in Australia
}

\author{
Emily Duncanson, ${ }^{1}$ Paul N Bennett, ${ }^{2}$ Andrea Viecelli, ${ }^{3}$ Kathryn Dansie, ${ }^{1}$ \\ William Handke, ${ }^{4}$ Allison Tong, ${ }^{5}$ Suetonia Palmer, ${ }^{6}$ Shilpanjali Jesudason, ${ }^{7,8}$ \\ Stephen P McDonald, ${ }^{1,7}$ Rachael L Morton (1) , ${ }^{9}$ On behalf of the Symptom \\ monitoring Wlth Feedback Trial (SWIFT) Investigators
}

To cite: Duncanson E, Bennett PN, Viecelli A, et al. Feasibility and acceptability of e-PROMs data capture and feedback among patients receiving haemodialysis in the Symptom monitoring With Feedback Trial (SWIFT) pilot: protocol for a qualitative study in Australia. BMJ Open 2020;10:e039014. doi:10.1136/ bmjopen-2020-039014

\section{- Prepublication history and} additional material for this paper are available online. To view these files, please visit the journal online (http://dx.doi. org/10.1136/bmjopen-2020039014).

Received 02 April 2020 Revised 22 August 2020 Accepted 11 October 2020

D) Check for updates

(c) Author(s) (or their employer(s)) 2020. Re-use permitted under CC BY-NC. No commercial re-use. See rights and permissions. Published by BMJ.

For numbered affiliations see end of article.

\section{Correspondence to} Professor Rachael L Morton; Rachael.morton@ctc.usyd. edu.au

\begin{abstract}
Introduction People receiving haemodialysis experience a high symptom burden and impaired quality of life. The use of patient-reported outcome measures (PROMs) is increasing in nephrology care, however their acceptability, utility and impacts are not well understood.

Methods and analysis We describe a protocol for a qualitative study to evaluate the feasibility and acceptability of electronic-PROMs (e-PROMs) data capture and feedback in haemodialysis following the pilot Symptom monitoring Wlth Feedback Trial (SWIFT). SWIFT involves linkage of e-PROMs data, including symptoms and health-related quality of life, to the Australia and New Zealand Dialysis and Transplant Registry with feedback to patients' treating nephrologists and nurse unit managers. Focus groups and semistructured interviews will be conducted with nephrologists ( $n=15)$, dialysis nurses $(n=24)$ and patients receiving haemodialysis $(n=24)$ from six dialysis units in Australia. Question topics will include the technical and clinical feasibility and acceptability of ePROMs reporting and feedback (including the barriers and enablers to uptake) and perceived impact on patient care and outcomes. Transcripts will be analysed thematically and guided by Normalisation Process Theory.

Ethics and dissemination Ethics approval was obtained from the relevant hospital Human Research Ethics Committees (HREC/18/CALHN/481; HREC/MML/54599).

The findings from the SWIFT pilot and qualitative evaluation will inform the implementation of the SWIFT main trial, and more broadly, the use of e-PROMs in clinical settings and registries.
\end{abstract}

Trial registration number ANZCTRN12618001976279.

\section{INTRODUCTION}

More than 3 million people worldwide receive treatment for kidney failure and numbers are expected to rise sharply due to population growth and increasing prevalence of diabetes
Strengths and limitations of this study

- Qualitative methods will obtain diverse, detailed and in-depth insights into the processes involved in electronic patient-reported outcome measures (ePROMs) integration in nephrology care.

- Barriers and enablers to the use of e-PROMs in clinical care, and perceived impacts on patient care and outcomes, as well as to health professionals will be explored alongside a 6 -month pilot study.

- Findings from this qualitative analysis will help refine the main Symptom monitoring With Feedback Trial, to improve acceptability and ease of delivery of the intervention.

Views and perspectives from a range of stakeholders including patients, nurses and nephrologists will be sought.

- Evaluation of the pilot study is limited to six nephrology centres in two states in Australia and therefore findings may not be transferable or identify issues applicable to all potential trial sites.

and cardiovascular comorbidities. ${ }^{1}$ Haemodialysis remains the most common treatment modality for kidney failure, including 10983 Australians and 1978 New Zealanders. ${ }^{2}$ While haemodialysis can prolong life, patients experience burdensome symptoms ${ }^{3-6}$ including pain, fatigue, itch, nausea, sleep difficulties and depression, ${ }^{3}$ that impair quality of life. Quality of life among people on facility-based haemodialysis, as measured by standard selfreport measures such as the EuroQOL 5 dimensions 5 levels (EQ-5D-5L), has shown to be around $59 \%$ of full health, with a large meta-analysis reporting a minimum of $42 \%$ of 
full health. ${ }^{5}$ This is lower than that among people with metastatic prostate cancer and of those with paralysis due to spinal cord injury. ${ }^{6}$

Studies have largely focused on biochemical markers of health and survival, while patient-important outcomes including symptom burden and quality of life are infrequently reported. ${ }^{7-9}$ In addition to measures of morbidity and mortality, dialysis adequacy based on clearance of small molecules, particularly urea, has been the biochemical marker of choice for researchers and dialysis clinicians. ${ }^{79}$ Notwithstanding the lack of an evidentiary basis for an association of biochemical markers with quality of life, these markers may be irrelevant to patients receiving dialysis as they do not fully capture their experiences and perspectives of health and care.

Patient-reported outcome measures (PROMs) assess outcomes that reflect how patients feel and function, as reported directly from the patient without interpretation from others. ${ }^{10}$ In primary care and oncology settings, collecting and providing PROMs to health professionals has shown benefits including identifying unmet patient physical and psychological needs, enhancing patientprovider communications and shared decision-making and improved quality of care. ${ }^{10}$ Findings from two randomised trials in oncology also suggest symptom monitoring improves patient quality of life and survival. ${ }^{11-13}$ There is a dearth of evidence on the systematic measurement of and response to symptom burden among people receiving dialysis, representing potential missed opportunities to improve patient care, quality of life and survival, particularly given the severity of symptoms for these patients.

A recent prospective cross-sectional survey of 79 dialysis units in Australia and New Zealand showed that 55 of 79 units $(70 \%)$ collected PROMs or patient-reported experience measures. ${ }^{14}$ The Integrated Palliative Outcome Scale-Renal (IPOS-Renal) was the most commonly reported instrument to measure symptoms $(40 \%$ of units) and the EQ-5D-5L for the assessment of quality of life $(24 \%)$. Insufficient time and staff resources were identified as the main barriers to collection of PROMs (19 out of 24 units $(79 \%)$ ).

The Australia and New Zealand Dialysis and Transplant Registry (ANZDATA) is a clinical quality registry established in 1977 in which clinical and laboratory data are held on people receiving dialysis or kidney transplantation (eg, mortality, comorbidities, treatments, dialysis adequacy). While clinical quality registries such as ANZDATA serve to assess the quality of clinical care in order to improve patient outcomes, they do not generally collect PROMs. ${ }^{15}$ Linkage of PROMs data with an already established clinical and treatment registry (ANZDATA) represents a method of combining data that is potentially meaningful to both health professionals and patients, including aspects of patient well-being and care.

In line with patient priorities, ${ }^{9}$ use of PROMs is being increasingly encouraged in nephrology, but knowledge on how to effectively implement PROMs collection to improve routine clinical practice is limited. One theory that can guide understanding of the required behaviour change to incorporate electronic PROMs collection into routine care, and take action based on these data, is Normalisation Process Theory (NPT). This theory aims to explain the factors needed for successful implementation and integration of interventions into routine work, and is easily applied to the use of technology in healthcare settings. Using NPT as a framework for analysis of qualitative data in a pilot study can improve the design of trials with a complex intervention by highlighting potential problems with recruitment or data collection. It also facilitates analysis of the potential benefits and harms of providing symptom information to clinicians and the impact of this data on patient care and outcomes.

In addition, the potential benefits and harms of providing symptom information to members of the patients' treating team on patient care and outcomes are unknown. The aim of the main Symptom monitoring WIth Feedback Trial (SWIFT) is to assess the clinical and cost-effectiveness of regular symptom monitoring in people receiving in-centre haemodialysis with feedback to clinicians, embedded within ANZDATA. In preparation for the main trial, the SWIFT pilot study is being conducted in six Australian dialysis centres in Queensland and South Australia to evaluate the technical and clinical feasibility and acceptability of this intervention. Here we describe the protocol for a qualitative study to evaluate the SWIFT pilot study. Following the completion of the SWIFT pilot study, focus groups and semistructured interviews with patients, nurses and nephrologists will specifically address the questions of how acceptable and feasible it is to collect, interpret and act on electronicPROMs (e-PROMs) including quality of life and symptom burden; and why (or why not) the provision of symptom information changes clinician-patient conversations and care. This study will also uncover the perceived impact of e-PROMS collection and feedback on patient care and outcomes, as described by those who are at the coalface of their delivery and use.

\section{METHODS AND ANALYSIS}

This study is informed by the UK Medical Research Council Guidance for Process Evaluation ${ }^{17}$ and will be reported in accordance with the Consolidated Criteria for Reporting Qualitative Research. ${ }^{18}$

\section{Context: SWIFT pilot study}

Setting

Four South Australian dialysis centres and two Queensland dialysis centres in Australia will participate in the SWIFT pilot study. The centres are of varying sizes, cover heterogeneous socioeconomic populations and geographical areas and have different models of care, reflecting realworld variations in practice and haemodialysis service delivery. At the time of the SWIFT pilot study, approximately 430 patients were receiving haemodialysis across 
these centres. Centre-based consent for the pilot was obtained with a patient-based opt-out option to minimise selection bias. PROMs data collection for the SWIFT pilot is scheduled to complete in April 2020.

\section{SWIFT intervention}

\section{Design}

Sites will be randomly allocated to either the intervention arm (3-month symptom monitoring with feedback to clinicians) or the non-intervention arm (quality of life collection only). E-PROMs will be collected via a tablet (Samsung Galaxy Tab A V.10.5) at patients' in-centre haemodialysis appointments either before, during or after dialysis. For all sites, quality of life will be measured using the EQ-5D-5L $\mathrm{L}^{19}$ at baseline and 6 months. For those in the intervention arm, symptom burden will be measured using the IPOS-Renal ${ }^{20}$ at baseline, 3 and 6 months. These instruments were chosen for several reasons including familiarity with their use as identified in our prior national audit, validation in Australian patients on dialysis, acceptability to patients and sensitivity to the changes in quality of life likely to be brought about through comprehensive symptom management. ${ }^{140-22}$

\section{Administration and data collection}

Nurse unit managers of participating sites will be provided Quick Response codes unique to each patient, a tablet and user guides with e-PROMs administration instructions. Clinical nurses involved in the patients' care will be responsible for preparing the tablet, providing it to patients and cleaning it after use according to local infection-control policies. Patients will be encouraged to complete the questions honestly and without input from others. All dialysis nursing staff will have attended 1-2 training sessions giving information about the study, the purpose of collecting PROMs, and for those in the intervention arm, the process for interpreting IPOS-Renal symptom scores.

\section{E-PROMs feedback}

E-PROMs data will be stored temporarily on the tablets and then transferred periodically to specific database tables in the ANZDATA Registry. Tailored emails containing the individual patient's IPOS-Renal scores will be generated and sent by ANZDATA staff to the dialysis nurse unit manager and to the patient's treating nephrologist, with patients reporting scores of 3 (severe symptoms) or 4 (overwhelming symptoms) highlighted in the body of the email. In this email, clinicians will be encouraged to discuss symptoms with their patients at their next clinical encounter and will be provided with an attached document including hyperlinks to evidence-based symptom management guidelines.

\section{Qualitative evaluation}

The schema of the qualitative evaluation of the SWIFT pilot is provided in figure 1. Qualitative methods will be used to elicit the experiences and perspectives of patients, nurse unit managers, nursing staff and nephrologists regarding the intervention process, acceptability and feasibility, and perceived impacts on patient care and outcomes. The findings will inform refinement of the intervention processes for the main SWIFT trial and reveal how this system may be implemented into routine care for people receiving haemodialysis.

\section{Participant selection and recruitment \\ Patients}

Eligible participants will be English-speaking patients or those with an interpreter available, $\geq 18$ years, who are able to provide informed consent and who completed the IPOS-Renal and/or the EQ-5D-5L at 6 months. A purposive sampling strategy will be applied to capture a diverse range of patient experiences and perspectives in focus groups and interviews, based on demographic (sex, age, ethnicity and linguistic background) and clinical factors (time since haemodialysis commencement and symptom burden severity). As interviews are being completed, we will monitor the demographic and clinical characteristics to ensure to target recruitment to include any 'missing' characteristics. Patients participating in the SWIFT pilot study will be offered information about the qualitative evaluation component by nursing staff, who will then

\section{Data Collection
(post 6-month e-PROMs collection)}

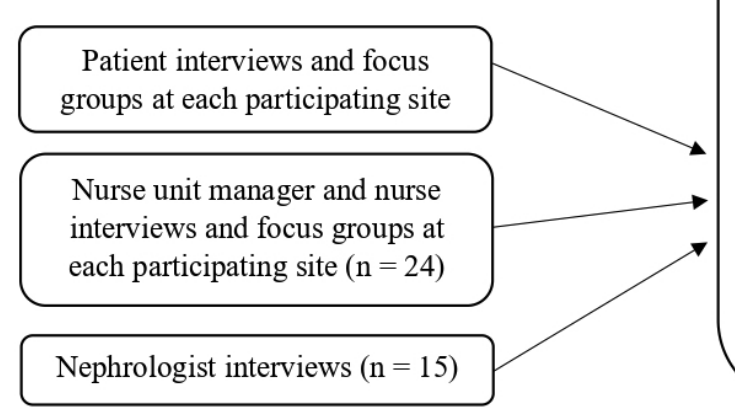

\section{Data Analysis}

Thematic analysis Identification of themes of the experience and perceptions of renal medical staff and patients regarding intervention process, acceptability and feasibility (guided by Normalisation Process Theory) and perceived impacts on patient care and outcomes

Figure 1 Qualitative evaluation study schema. e-PROMs, electronic-patient-reported outcome measures; SWIFT, Symptom monitoring WIth Feedback Trial. 
put forward interested patients to the research team. A member of the research team will contact potential participants, explain the purpose of the interview or focus group and invite them to participate. It will be emphasised that participation is voluntary and that participants have the right to withdraw at any time without having to provide a reason. Those who express interest will be provided a participant information sheet and consent form specific to the qualitative study and then followed up for consenting and formal arrangement of the interview or focus group.

\section{Nurse unit managers and nurses}

Nurse unit managers and nursing staff at participating sites will be eligible to participate in a semistructured interview or focus group. All dialysis nursing staff will be informed of the qualitative component of the SWIFT pilot study and those interested will be put forward to the research team by the nurse unit manager. Purposive sampling will ensure nurses of varying ages with different roles and experience and from both arms of the trial are captured. Views of nurses not involved in administration of e-PROMs in their unit will also be sought, to also understand symptom management practices in standard care. Standard consenting processes as described above, will be followed.

\section{Nephrologists}

Nephrologists eligible to participate in an interview will be those with patients in either study arm, as well as nephrologists not participating in the pilot study from an additional centre in South Australia, to explore processes and practices of symptom management in standard care. Purposive sampling will ensure nephrologists of diverse age, sex, level of experience and practice site are included. Nephrologists of participating sites in the SWIFT pilot study who received e-PROMs data indicating severe or overwhelming symptoms in their patients, as well as those who did not, will be interviewed to gather diverse perspectives. A member of the research team will contact nephrologists, explain the purposes of the interview and invite them to participate. Standard consenting processes as described above, will be followed. Patients will be reimbursed with a gift card to the value of $\$ 50$. No reimbursement will be provided to health professionals.

\section{Data collection}

Interviews and focus groups with patients, nurses and nephrologists are scheduled to commence in April 2020 and completed by July 2020 within approximately 4 weeks of centres completing the 6-month data collection. This timeframe was believed to be critical to ensuring participants' accurate recall of their experiences in the trial and allowing opportunity for patients of sites in the intervention arm of the pilot to have had contact with their treating nephrologist, for potential actioning of symptom data. Data collection and analysis will involve an interactive process, whereby initial transcripts will be reviewed by other members of the research team, and modifications and additions to some questions made. We anticipate analysis of qualitative data will be completed by October 2020.

Interviews and focus groups with patients on haemodialysis and nursing staff

Authors ED, AV, PB and KD will conduct separate focus groups or semistructured interviews with patients or nurses at each participating site. Focus groups will aim to have six to eight participants in each and last approximately $90 \mathrm{~min}$. This method of data collection was selected as an option as it is expected to provide opportunity to harness group dynamics (eg, brainstorming, clarification of ideas and differences of opinion) for detailed discussion among patients or nursing staff. Focus groups will take place in a private room at the hospital or dialysis unit. A tablet with sample versions of the EQ-5D-5L and IPOS-Renal surveys will be available during focus groups and interviews to aid discussion, if necessary. If a patient or nurse is unable to attend a focus group, they will have the opportunity of participating in a semistructured interview with a member of the research team, in person or via telephone. Semistructured question guides for patient and nurse interviews and focus groups are provided in online supplemental files 1 and 2). These include some set/specific questioning by the researcher regarding the feasibility and acceptability topics of interest, as well as opportunity for questions and prompts that are guided by participants' responses. These were informed by a literature review of previous research on PROMs collection ${ }^{23}$ and discussion among the multidisciplinary research team, including nephrologists, nurses and consumers.

Prior to the commencement of focus groups and interviews, participants will be reminded of the aim of the project and their right to withdraw at any time. Interviews and focus groups will not commence until participants' written informed consent is obtained. They will be audio recorded with participants' consent and transcribed verbatim. Participants will be made aware that only researchers involved in the qualitative evaluation of SWIFT will have access to their transcripts and that de-identified versions and exemplar quotes will be viewed by other project staff and will form the basis of publications and presentations.

\section{Interviews with nephrologists}

Author AV will conduct semistructured interviews with nephrologists either via telephone or face-to-face at a mutually convenient time and location (eg, office or meeting room in the hospital or dialysis units). The interview guide is provided in online supplemental file 3) and was informed by relevant literature and discussion among the research team. Interviews will be audio recorded and transcribed verbatim. Interviews will not commence until participants' written informed consent is obtained.

One strength of this pilot study is the sampling of the three stakeholder groups: patients, nurses and 
nephrologists. These methods of interview and focus groups will provide sufficient data to elicit their experiences and perspectives regarding the intervention process, acceptability and feasibility, and perceived impacts on patient care and outcomes in order to inform the main trial. We anticipate this will require at least 20 participants in total, 10-12 from the intervention group and $8-10$ from the control group.

\section{Data analysis}

The data will be entered into HyperRESEARCH software and analysed thematically. ${ }^{24}$ Thematic analysis enables the identification and analysis of themes across qualitative data, to reveal implicit meaning and phenomena in relation to the research question. Identification of themes will be guided by NPT, an analytic framework which can explain how technology is implemented, embedded and integrated into healthcare settings, including factors that promote or inhibit 'normalisation' into practice..$^{25} \mathrm{NPT}$ consists of four constructs:

1. Coherence: how people individually and collectively understand and make sense of implementing a new practice.

2. Cognitive participation: how people build and sustain engagement with new practice.

3. Collective action: how people enact the new practice.

4. Reflexive monitoring: how people formally and informally appraise and understand the effects of the new practice once in use. ${ }^{26}$

All transcripts will first be coded inductively by two researchers, including the identification of the perceived impacts of e-PROMS collection and feedback on patient care and outcomes. NPT will then be used as a guide to deductively identify additional/alternative themes, to examine how e-PROMs become 'normalised' into dialysis units, including the barriers and facilitators. Codes will be grouped into themes and subthemes and relationships among them identified. Data of each participant group (patients, nurses, nephrologists) will be analysed separately until data saturation. Derived themes will be reviewed by other members of the research team throughout the analysis process as researcher triangulation, to ensure the full range and depth of data are reflected in the findings. ${ }^{18}$ Comparisons will be drawn between nurse, nephrologist and patient responses. A summary of themes will be provided to participants for comment and feedback (member checking) to ensure their views are captured and accurately reflected in final analysis and reporting of results.

\section{Patient and public involvement}

Prior to the pilot, two patients in a local haemodialysis unit completed the e-PROMs while undergoing dialysis and were observed by two members of the research team who took note of relevant environmental factors, use of tablet stands/cases and patient-nurse interactions. A consumer partner (carer of person receiving haemodialysis) and member of the SWIFT Management Committee provided input throughout the development of the project design and qualitative study protocol, including to the interview and focus group question guides. A group of consumers from participating pilot sites will have opportunity to review and interpret findings of the qualitative study, create patient-version summaries of these and assist in refining the intervention and design of the SWIFT main trial. The Better Evidence And Translation-Chronic Kidney Disease 'Consumers in Research Programme' will train the consumers, with additional support provided through the Australian Clinical Trials Alliance Consumer and Community Involvement workshop (https://clinical trialsalliance.org. au/events-forums/consumer-community-involvement-in-clinical-trials-acta-training-workshop$3 /)$. We will also provide specific individual training and mentorship in the analysis of qualitative data by qualitative researchers on the investigator team.

\section{DISCUSSION}

This study aims to gain rich and in-depth insights from key stakeholders about the SWIFT intervention processes, acceptability and feasibility, and impact on patient care and outcomes. To ensure the successful implementation of an e-PROMs system and use of patient data, understanding factors such as resourcing, technological issues, timing and setting of data collection and appropriate feedback mechanisms, from the perspectives of renal medical staff and patients, will be imperative. Themes identified from the qualitative evaluation of the SWIFT pilot will inform and refine the intervention for the main trial, based on the views of those providing and receiving clinical care.

From the patient perspective, the feasibility of various data collection timepoints will be important to explore (ie, before, during or after a dialysis session). Surroundings and privacy in the dialysis unit, presence of blood lines and patients' handedness need to be considered. Patient confidence with electronic devices and their experience completing the e-PROMs tools will be explored to understand the user experience with the device, including its logic, item presentation and navigation prompts. The timing of data collection also raises the question of how the patient's state and symptoms at the time may influence reports and consideration of the prescribed recall periods in surveys. Interviews and focus groups will also reveal patients' views and experiences of changes (or not) in clinical care and management based on feedback of results to their renal team and perceived impacts on quality of life.

The views of dialysis nurse unit managers and nursing staff will be important to understand uptake of the data collection process, resourcing requirements and administration and information technology (IT) issues associated with e-PROMs collection, 'on the ground' in dialysis units. Staff familiarity and confidence with electronic devices may be factors that influence uptake and implementation. Nurse interviews and focus groups will 
explore IT aspects, staff support needs and potential solutions based on their experiences. To mitigate challenges that may hinder the implementation of e-PROMs collection, IT issues such as method of patient identification and internet availability in dialysis units need to be understood. Moreover, patient-reported outcomes may be influenced by how nurses administer the data collection tools, deliver instructions and provide assistance (or not) to patients during reporting.

A successful feedback mechanism is imperative to the usefulness of collected PROMs to improving patient care and outcomes. A feedback process includes identifying an optimal mode of notification and communication of results to clinical teams (eg, email, printed data inserted into clinical notes in dialysis unit or a patientinitiated log-in system) and timing of delivery of patientreported outcomes to clinicians to ensure accessibility and relevance at the patient's next clinical encounter and opportunity for timely clinical action. Preferences for these are likely to be influenced by complex system and operational differences across hospital and dialysis units. It is also important to explore clinician preferences for the presentation of results (eg, providing all of a patient's symptom scores, or only those identified as burdensome) and how PROMs collection may affect or be affected by centres' existing procedures for responding to problematic symptoms as part of standard care. We acknowledge that our data collected on implementation of e-PROMs capture and feedback within the context of a randomised trial, may not necessarily reflect routine care.

While haemodialysis maintains life, patients often live with substantial physical and psychosocial symptom burden. Consistent with the increased emphasis on patient-centred care, SWIFT will address patientprioritised issues and outcomes that have previously been overlooked. Qualitative evaluation of the SWIFT pilot study will provide invaluable insights from those ultimately responsible for the implementation and use of e-PROMs and will inform a large multicentre registrybased randomised controlled trial to evaluate whether symptom monitoring with feedback improves care and outcomes for people receiving haemodialysis.

\section{ETHICS AND DISSEMINATION}

Ethics approval for SWIFT was obtained from the relevant hospital Human Research Ethics Committees (HREC/18/CALHN/481; HREC/MML/54599). Findings from the SWIFT pilot will be presented at scientific meetings and prepared for publication in peer-reviewed journals. Participating units will be provided a summary of the findings, including patient-facing versions co-developed with consumers.

\section{Author affiliations}

${ }^{1}$ Australia and New Zealand Dialysis and Transplant (ANZDATA) Registry, South Australian Health and Medical Research Institute, Adelaide, South Australia, Australia
${ }^{2}$ Faculty of Health Medicine Nursing and Behavioural Sciences, Deakin University, Burwood, Victoria, Australia

${ }^{3}$ School of Medicine, Princess Alexandra Hospital, Woolloongabba, Queensland, Australia

${ }^{4}$ Consumer representative, Private citizen, Canberra, ACT, Australia

${ }^{5}$ Sydney School of Public Health, The University of Sydney, Sydney, New South Wales, Australia

${ }^{6}$ Department of Medicine, University of Otago, Christchurch, New Zealand ${ }^{7}$ Central and Northern Adelaide Renal and Transplantation Services (CNARTS), Royal Adelaide Hospital, Adelaide, South Australia, Australia

${ }^{8}$ Faculty of Health and Medical Sciences, University of Adelaide, Adelaide, South Australia, Australia

${ }^{9}$ NHMRC Clinical Trials Centre, The University of Sydney, Camperdown, New South Wales, Australia

Twitter Allison Tong @allisontong1 and Rachael L Morton @RachaeIMorton12

Acknowledgements We thank Portia Westall, Rebecca Mister, Sarah Mulray, Helen Pater, Veronia Oliver, Kylee McCarthy, Stephen Abela, Ashleigh Jaensch, Lynn Phillips, Kath Hampel and Chris Kelly for their support in the conduct of the SWIFT pilot study.

Collaborators David Harris, David Johnston, Carmel Hawley, Chris Brown, John Simes, Liliana Laranjo, Rebecca Mister, Portia Westall, Enrico Coiera, Matthew Jose, Fergus Caskey and Cecile Couchoud as the Symptom monitoring Wlth Feedback Trial (SWIFT) investigators.

Contributors RLM is the chief investigator of the SWIFT pilot study. She conceived and designed the study, drafted the manuscript and reviewed the final version. ED, $\mathrm{PB}, \mathrm{AV}$ and $\mathrm{KD}$ designed the semistructured interview guide, drafted the manuscript and reviewed the final version. WH provided consumer input into the choice of instruments, participant information and consent forms, and reviewed the final draft. AT provided input on methodological aspects of the study design and analysis. SP, SJ and SPM designed the study, drafted the manuscript and reviewed the final version. The final draft was approved by all authors and co-investigators.

Funding SWIFT is supported by a Kidney Health Australia Medical and Scientific Research Funding Grant (KHA2018-RM), a National Health and Medical Research Council (NHMRC) Project Grant (APP1159051), and BEAT-CKD Programme Grant (APP1092957). Author RLM is supported by an NHMRC TRIP Fellowship (APP1150989) and a University of Sydney, Robinson Fellowship. Author AV is supported by a Princess Alexandra Research Foundation Research Grant and a Jacquot Research Establishment Fellowship.

Competing interests None declared.

Patient consent for publication Not required.

Provenance and peer review Not commissioned; externally peer reviewed.

Supplemental material This content has been supplied by the author(s). It has not been vetted by BMJ Publishing Group Limited (BMJ) and may not have been peer-reviewed. Any opinions or recommendations discussed are solely those of the author(s) and are not endorsed by BMJ. BMJ disclaims all liability and responsibility arising from any reliance placed on the content. Where the content includes any translated material, BMJ does not warrant the accuracy and reliability of the translations (including but not limited to local regulations, clinical guidelines, terminology, drug names and drug dosages), and is not responsible for any error and/or omissions arising from translation and adaptation or otherwise.

Open access This is an open access article distributed in accordance with the Creative Commons Attribution Non Commercial (CC BY-NC 4.0) license, which permits others to distribute, remix, adapt, build upon this work non-commercially, and license their derivative works on different terms, provided the original work is properly cited, appropriate credit is given, any changes made indicated, and the use is non-commercial. See: http://creativecommons.org/licenses/by-nc/4.0/.

ORCID iD

Rachael L Morton http://orcid.org/0000-0001-7834-0572

\section{REFERENCES}

1 Liyanage T, Ninomiya T, Jha V, et al. Worldwide access to treatment for end-stage kidney disease: a systematic review. Lancet 2015;385:1975-82. 
2 Registry A. Chapter 2: Prevalence of Renal Replacement Therapy for End Stage Kidney Disease. In: ANZDATA 42nd annual report 2019. Adelaide, Australia: Australia and New Zealand Dialysis and Transplant Registry, 2019.

3 Almutary $\mathrm{H}$, Bonner A, Douglas $\mathrm{C}$. Which patients with chronic kidney disease have the greatest symptom burden? A comparative study of advanced CKD stage and dialysis modality. J Ren Care 2016;42:73-82.

4 Davison SN, Levin A, Moss AH, et al. Executive summary of the KDIGO controversies conference on supportive care in chronic kidney disease: developing a roadmap to improving quality care. Kidney Int 2015;88:447-59.

5 Wyld M, Morton RL, Hayen A, et al. A systematic review and meta-analysis of Utility-Based quality of life in chronic kidney disease treatments (Utility-Based quality of life in CKD). PLoS Med 2012;9:e1001307.

6 Morton RL. Quality of life in chronic kidney disease. Heidelberg: Springer, 2014.

7 Evangelidis N, Tong A, Manns B, et al. Developing a set of core outcomes for trials in hemodialysis: an international Delphi survey. Am J Kidney Dis 2017;70:464-75.

8 Sautenet B, Tong A, Williams G, et al. Scope and consistency of outcomes reported in randomized trials conducted in adults receiving hemodialysis: a systematic review. Am J Kidney Dis 2018;72:62-74.

9 Tong A, Manns B, Hemmelgarn B, et al. Establishing core outcome domains in hemodialysis: report of the standardized outcomes in Nephrology-Hemodialysis (SONG-HD) consensus workshop. Am J Kidney Dis 2017;69:97-107.

10 Valderas JM, Kotzeva A, Espallargues M, et al. The impact of measuring patient-reported outcomes in clinical practice: a systematic review of the literature. Qual Life Res 2008;17:179-93.

11 Basch E, Deal AM, Dueck AC, et al. Overall survival results of a trial assessing patient-reported outcomes for symptom monitoring during routine cancer treatment. JAMA 2017;318:197-8.

12 Basch E, Deal AM, Kris MG, et al. Symptom monitoring with patientreported outcomes during routine cancer treatment: a randomized controlled trial. J Clin Oncol 2016;34:557-65.

13 Velikova G, Booth L, Smith AB, et al. Measuring quality of life in routine oncology practice improves communication and patient well-being: a randomized controlled trial. J Clin Oncol 2004;22:714-24
14 Morton RL, Lioufas N, Dansie K, et al. Use of patient-reported outcome measures and patient-reported experience measures in renal units in Australia and New Zealand: a cross-sectional survey study. Nephrology 2020;25:14-21.

15 (USRDS) TUSRDS. United States renal data system Minneapolis, 2019. Available: https://www.usrds.org/Default.aspx

16 Association RotERA-EDaT. ERA-EDTA Regsitry, 2019. Available: https://era-edta-reg.org/index.jsp?p=1 [Accessed 3 Feb 2020].

17 Moore GF, Audrey S, Barker M, et al. Process evaluation of complex interventions: medical Research Council guidance. BMJ 2015;350:h1258.

18 Tong A, Sainsbury P, Craig J. Consolidated criteria for reporting qualitative research (COREQ): a 32-item checklist for interviews and focus groups. Int J Qual Health Care 2007;19:349-57.

19 Foundation ER. EQ-5D-5L: EuroQol research Foundation, 2020. Available: https://euroqol.org/eq-5d-instruments/eq-5d-5l-about/ [Accessed 13 Feb 2020].

20 Raj R, Ahuja K, Frandsen M, et al. Validation of the IPOS-Renal symptom survey in advanced kidney disease: a cross-sectional study. J Pain Symptom Manage 2018;56:281-7.

21 Lowney AC, Myles HT, Bristowe K, et al. Understanding what influences the health-related quality of life of hemodialysis patients: a collaborative study in England and ireland. J Pain Symptom Manage 2015;50:778-85

22 Breckenridge K, Bekker HL, Gibbons E, et al. How to routinely collect data on patient-reported outcome and experience measures in renal registries in Europe: an expert consensus meeting. Nephrol Dial Transplant 2015;30:1605-14.

23 Anderson NE, Calvert M, Cockwell P, et al. Using patient-reported outcome measures (PROMs) to promote quality of care in the management of patients with established kidney disease requiring treatment with haemodialysis in the UK (PROM-HD): a qualitative study protocol. BMJ Open 2018;8:e021532.

24 Braun V, Clarke V. Using thematic analysis in psychology. Qual Res Psychol 2006;3:77-101.

25 McEvoy R, Ballini L, Maltoni S, et al. A qualitative systematic review of studies using the normalization process theory to research implementation processes. Implement Sci 2014;9:1-13.

26 Jones CHD, Glogowska M, Locock L, et al. Embedding new technologies in practice - a normalization process theory study of point of care testing. BMC Health Serv Res 2016;16:591. 